\title{
Computational Study of Scattering Elastic Waves Due to a Teredo Marine Borer-Like Cylindrical Defect Embedded in an Isotropic Solid Cylinder
}

\author{
Ahmed Murgab Mohammed Mahila, ${ }^{a}$, Wing Kong Chiu ${ }^{b}$ and Benjamin Vien ${ }^{c}$ \\ Department of Mechanical and Aerospace Engineering, Monash University, Wellington Rd, \\ Clayton, VIC 3800, Australia \\ aahmed.mohammedmahil@monash.edu, b wing.kong.chiu@monash.edu, \\ cben.vien@monash.edu
}

Keywords: Bulk Waves, Scattered Field, Finite Element Analysis, Group Velocity, Cylindrical Defect

\begin{abstract}
This paper showcases a quantitative investigation of scattering of ultrasonic waves experiences when impinging on a cylindrical defect inside a solid cylinder. Such cylindrical bores reduce the structural capacity of the cylinder, these defects constitute an even greater risk as they cannot be observed from the surface. The focal point investigated herein is to develop a better understanding of the wave's scattering when interacting with defects of cylindrical bore, mimicking the Teredo marine borer, within the solid cylinder. Two-dimensional Finite Element simulations are carried out using ABAQUS software. A $200 \mathrm{kHz} 5.5$ cycle Hann windowed excitation on an isotropic cylinder is simulated a point source excitation at the circumference of the cylinder is used. The scattering wave fields from a range of defect diameters through the solid cylinder are presented. Using Two-Dimensional Fast Fourier Transform, the wave mode and velocity of the scattered wavefield along various directions was identified in cylindrical coordinates, to decouple the wave modes. Computational results are presented for the scattering pattern as a function of cylindrical bore diameter size relative to wavelength. This study serves as an efficient approach when choosing an input for ultrasonic imaging, with the aim to obtain high fidelity imaging resolution for structural health monitoring applications.

\section{Introduction}

Marine borers especially the oyster family Teredo, also known as shipworm, have been known to cause wooden piles to lose their structural adequacy[1, 2]. The main problem with detecting these borers is that they burrow within the piles without leaving any distinguishable marks on the pile's surface which makes it hard to detect their existence by current methods. The focal point investigated in this paper is to develop a better understanding of the stress wave's scattering when interacting with defects of cylindrical bore shape within the solid cylinder. The defects mimic the Teredo marine borers that burrow in gun-barrel wooden structural piles Fig 1.
\end{abstract}

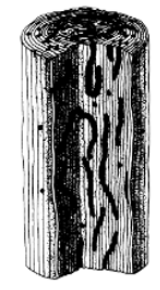

Figure 1. Teredo borers inside a gun-barrel wooden pile illustration [3]. 
Current methods to identify Teredo defects are laborious and ineffective in early detection of the marine borers such as quasi non-destructive testing using resistograph. [4]. Other methods such as X-ray scanner radiographs are hazardous, expensive and qualitative in nature $[5,6]$ and they are considered complementary methods to other mechanical and acoustic approaches. The most used non-destructive testing (NDT) method currently used is broadband stress waves using a hammer and ultrasonic receivers, this method depends on the time-of-flight (TOF) of the wave to determine the defect existence. In order to roughly learn about the defect location when using broadband impulses, iterative methods such as Algebraic Reconstruction Technique are utilised [7]. The issue with this method is that it does not assist in characterising the geometry of the defects or produce high fidelity images. To be able to deduce such information scattered wave field analysis are incumbent as well as the input signal's type.

In terms of the geometrical fundamentals of the Teredo defect, it can be approximated as a cylinder. Early work such as [8,9] investigated hollow cylinders embedded in a solid infinite isotropic medium as a scatterer and laid the formulation of plane waves scattering for compressional/longitudinal (P) and shear (S) waves experiencing mode conversion, while Lewis et al [10] generalised the formulations for a solid cylindrical scatterer. Furthermore, in composites [11] studied scattering for single modes by a cylindrical fibre and [12] developed a theoretical framework to study multiferroic composites that consist of randomly distributed fibres. Stress wave propagation in anisotropic wood formulations was investigated by [13]. Yan et al[14] used TOF to assess damage in timber poles using broadband excitation using a hammer. Scattered wave directivity patterns for a range of cylindrical defects were investigated in $[15,16]$ as a function of the wave number and cylindrical defect diameter. TOF is utilised in most of current literature as a primary method of detecting defects in wood using stress waves. However, TOF does not extract all the wavefield information and is limited to estimating the wavefield velocity. In order to achieve early detection of Teredo size defects inside a gun barrel/cylindrical wave guide it is incumbent to conduct a quantitative scatter analysis of the geometrical range for the Teredo Navalis from $5 \mathrm{~mm}$ up to $10 \mathrm{~mm}[3,17]$.

This paper computationally examines a hollow cylinder inside a solid isotropic cylinder and its interactions with elastic bulk waves, for a range of diameters focusing on the scatterer geometry. Despite wood being anisotropic and orthotropic, in this study the scattered wavefield is simulated in isotropic media. This will work as a reference for specific scatterer geometry behaviour in a well-known material. The wave modes, scattered wave patterns and their corresponding amplitudes are reported.

\section{Computational Procedure}

In this paper, numerical simulations using explicit dynamic finite element analysis tool in the ABAQUS software are presented. A cross-section of a solid cylinder of $200 \mathrm{~mm}$ in diameter is modelled in 2D plane strain for simulating bulk elastic wave propagating inside it. The material set as an isotropic aluminium (density of $2.7 \mathrm{~kg} / \mathrm{m}^{3}$, Possion's ratio of 0.33 and Young's modulus of $69 \mathrm{MPa}$ ). The isotropic material 4-node bilinear plane strain elements (CPE4R) were used to mesh the solid cylinder cross-section. In order to decouple wave modes propagating inside the cylinder's bulk the simulation data was converted from cartesian coordinates to polar coordinates in the $2 \mathrm{D}$ plane strain simulations with the centre of the defect is set as the point of origin. A narrow-band tone-burst signal carrying a central frequency is used as standard practice for the interpretation of the output signals [18]. For the simulation, the force excitation signal is a 5.5cycle Hann windowed with a centre frequency of $200 \mathrm{kHz}$. This loading configuration excites both $\mathrm{P}$ and $\mathrm{S}$ wave modes in the plane, however dominantly $\mathrm{P}$-wave impinges the defect. 


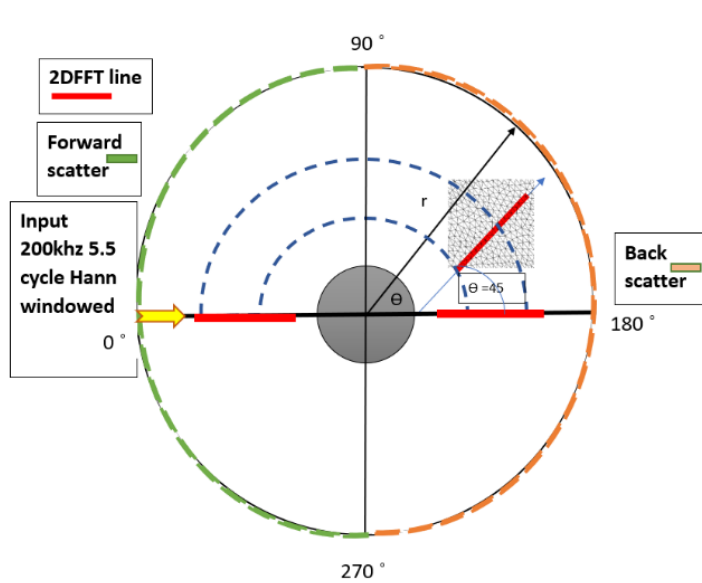

(a)

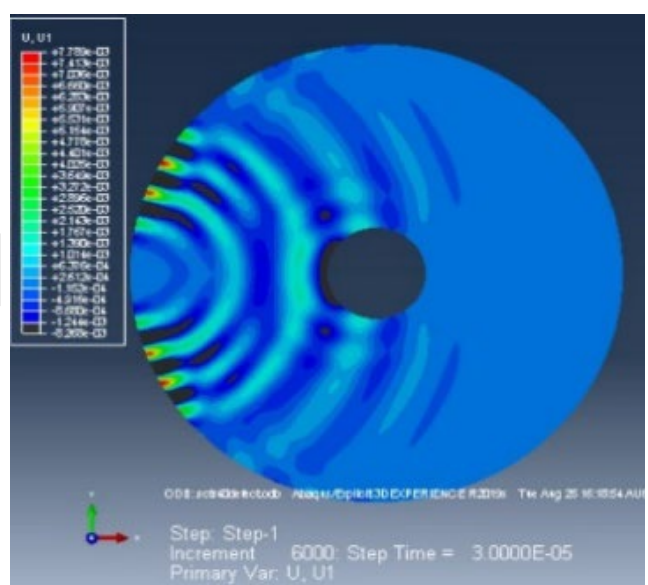

(b)

Figure 2. (a) Schematic diagram indicating location of defect and showing the 2D FFT line, excitation point and scattered wave measurements (b). $2 D$ FEA simulations of wave propagation at $30 \mu \mathrm{s}$

The defect is located in the middle of the cylinder as shown in Fig 2. The cylindrical crosssection is discretised into $0.2 \mathrm{~mm}$ 4-node bilinear plane strain elements. In Explicit analysis, the element size was set at $0.2 \mathrm{~mm}$, which satisfies the spatial requirement to capture ultrasonic wave propagation [19] and the time interval was set at 5ns to ensure the Courant Lewy Condition (CFL) [20] is satisfied, and meets the ABAQUS Explicit Time Integration stability limit of $0.6 \mathrm{~L} / \mathrm{C}$, where $\mathrm{L}$ denotes smallest element length and $\mathrm{C}$ the maximum wave velocity [21]. The defect is modelled as an infinite cylindrical defect with a maximum spacing of $0.2 \mathrm{~mm}$ around the circumference. The dependence of scattered amplitude with respect to defect diameter is investigated by varying the diameter size. The defect diameters (d) considered are of size from $5 \mathrm{~mm}$ to $10 \mathrm{~mm}$ diameter with $1 \mathrm{~mm}$ increment and then from $10 \mathrm{~mm}$ to $60 \mathrm{~mm}$ diameter with $10 \mathrm{~mm}$ increment, in order to investigate the effects when defect diameter is larger than the wavelength $\lambda \leq \mathrm{d}$ and smaller $(\lambda)>\mathrm{d}$ with respect to an incident $\mathrm{P}$-wave. At a centre frequency of $200 \mathrm{kHz}$, the wavelength for the $\mathrm{P}$ wave, $\lambda_{\mathrm{P}}$, is $31.6 \mathrm{~mm}$ and for $\mathrm{S}$ wave, $\lambda_{\mathrm{s}}$, is $15.8 \mathrm{~mm}$. Arrays of sensors were arranged in a straight equidistant line at different angles emerging from the excitation source and at an angle from the defect far side edge. It should be noted that, for $\lambda \leq \mathrm{d}$, the scattering contribution from the incident $\mathrm{S}$ waves are also present.

In order to investigate and verify the behaviour of the bulk waves ( $\mathrm{P}$ and $\mathrm{S}$ waves), the simulations data were processed using two-dimensional fast Fourier transformation (2D FFT); with node sensors at arrays radially away from the source of excitation. 2D FFT is performed on the nodes along lines at $0^{\circ}$ and $45^{\circ}$ from the defect, as indicated in Fig 2 . This is used to identify the dominant bulk wave mode from the 2D FFT, spatial distance is taken at least $1.5 \lambda$ away from the defect edge and over approximately $2 \lambda$ distance with zero padding [22].

The scattered wave field is extracted by subtracting the wave field of the defect simulation from the pristine simulation refer to Eq.1. Furthermore, in order to identify the scatter field modes, 2D FFT is implemented on scattered field data.

$$
U_{\text {scatter }}(\theta, r, t)=U_{\text {defect }}(\theta, r, t)-U_{\text {pristine }}(\theta, r, t)
$$


The scattered wave displacement is divided into two regions; back and forward scatter fields. They are defined in regions of scatter angle $270^{\circ}-90^{\circ}$ and $90^{\circ}-270^{\circ}$ as back and forward respectively. In Fig 6 , the way the directivity is obtained is by taking the maximum peak of the envelope of the time signal via the absolute Hilbert transformation, which was performed over the time domain signals measured at points on the circumference away from the defect in the farfield. In this paper time gating is conducted to validate the wave train of the scattered wave modes. Where scattered $\mathrm{P}$ wave arrives approximately after $35 \mu$ s the scattered $\mathrm{S}$ wave arrive at $50 \mu \mathrm{s}$. All the nodes on the circumference were at increments of $0.5^{\circ}$ from $0^{\circ}$ to $360^{\circ}$ and were used to obtain the polar directivity plots. The scattered wave amplitudes were measured at the cylinder circumference. Thus, the backward-scattered amplitudes were measured at $270^{\circ}$ to $90^{\circ}$ and the forward-scattered were measured at $90^{\circ}$ to $270^{\circ}$.

\section{Result}

Simulations were conducted to assess the effect of the defects on the incident wave and to detect mode conversion through 2D FFT results which were superimposed on the analytical wave mode velocities.

\section{Effect of defect diameter}

The 2D FFT results demonstrate the same pattern of mode conversion regardless of the circular defect sizes, indicating that mode conversion is independent of the defect diameter for the investigated range. From the FE simulations, $\mathrm{P}$ and S-waves were observed as depicted in Fig 3. At the $45^{\circ}$ sensor array, behind the defect as shown in Fig 3. P mode is dominant in radial ( $\mathrm{U}_{\mathrm{r}}$ ) direction as for the angular $\left(\mathrm{U}_{\theta}\right)$ direction, we observe a dominant $\mathrm{S}$ mode with a very weak $\mathrm{P}$ mode. It is clear that the 2D FFT velocity is in good agreement with the theoretical velocities at $200 \mathrm{kHz}$ and $400 \mathrm{rad} / \mathrm{m}$ wavenumber which is calculated by the relation $c=\frac{2 \pi}{k} f$ which yields $\mathrm{S}$ wave at $3155.185 \mathrm{~m} / \mathrm{s}$ at an agreement with the theoretical velocity $3160 \mathrm{~m} / \mathrm{s}$ as in [23]. The 2D FFT results are slightly skewed when compared to the theoretical lines in Fig 3 due to the angle of the sensor array. In Fig 4, at $0^{\circ}$ angle sensor's array before the defect, the P mode is observed in the $\mathrm{U}_{\mathrm{r}}$ direction, while in the $\mathrm{U}_{\theta}$ direction the $\mathrm{S}$ mode is present along with a faint $\mathrm{P}$ mode. Using polar coordinates helped decouple the wave modes. In Fig 5, at $0^{\circ}$ angle sensor's array after the defect we see a dominant $\mathrm{P}$ mode in $\mathrm{U}_{\mathrm{r}}$ in $\mathrm{U}_{\theta}$ an $\mathrm{S}$ mode with leaking $\mathrm{P}$ mode. These results demonstrate the incident wave mode conversion due to interaction with the defect. The observed mode conversion are the same upon varying the defect diameter.

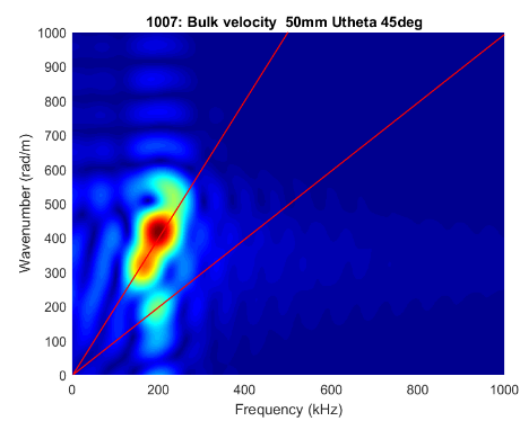

(a)

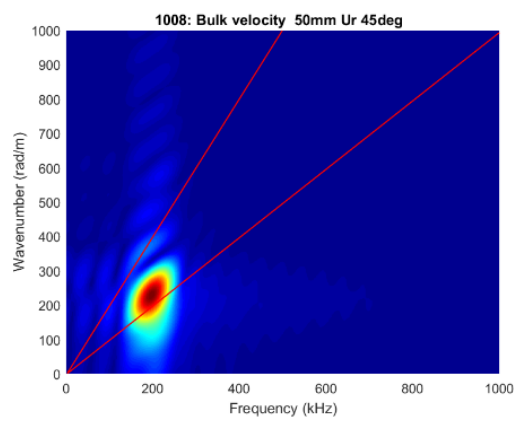

(b)

Figure 3. Analytical bulk velocities (P-S) vs $2 D$ FFT for scattered wavefield for the sensor array at $45^{\circ}$ from defect edge (a) $S$-wave and weak $P$-wave $U_{\theta}$ direction (b) $P$-wave $U_{r}$ direction 


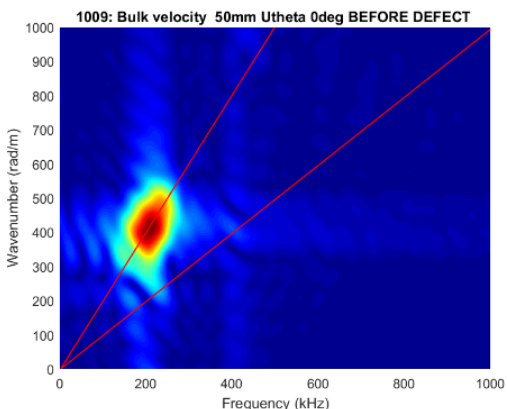

(a)

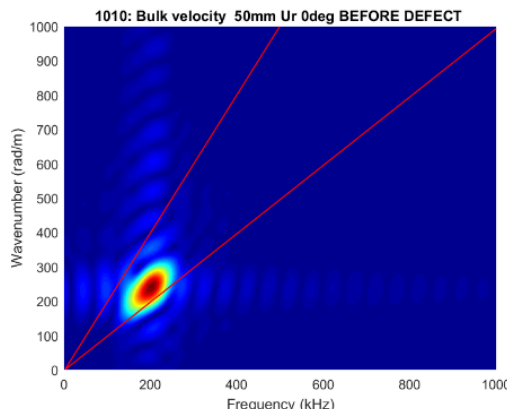

(b)

Figure 4. Analytical bulk velocities (P-S) vs 2D FFT for scattered wavefield for the sensor array at $0^{\circ}$ from defect edge (a) $S$-wave $U_{\theta}$ direction (b) $P$-wave $U_{r}$ direction

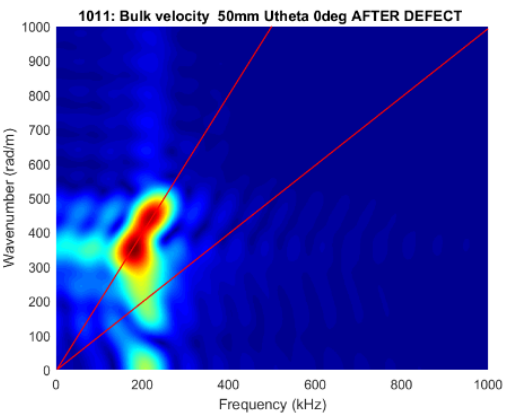

(a)

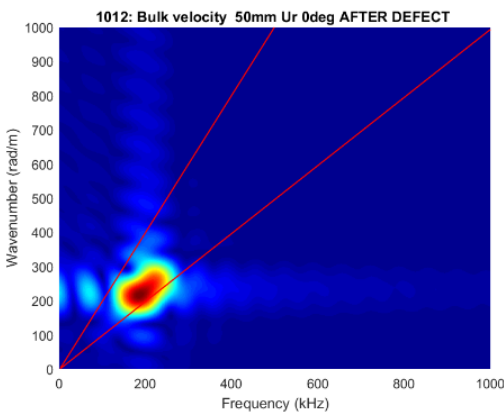

(b)

Figure 5. Analytical bulk velocities (P-S) vs 2D FFT for scattered wavefield for the sensor array at $180^{\circ}$ from defect edge (a) $S$-wave and weak $P$-wave $U_{\theta}$ direction (b) $P$-wave $U_{r}$ direction

$\mathrm{Ur}$

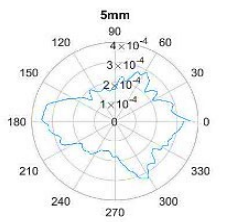

$\mathrm{U} \theta$
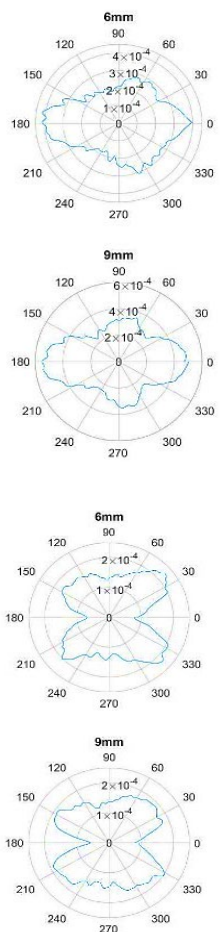
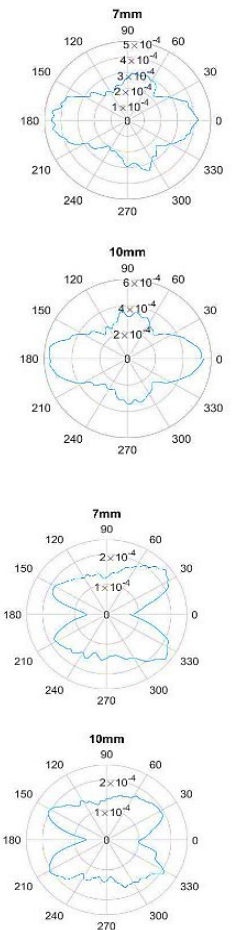

Figure 6. Scattered field amplitude directivity pattern in $U_{r}$ direction top and $U_{\theta}$ bottom for $d=$ (5mm to $10 \mathrm{~mm})$ 

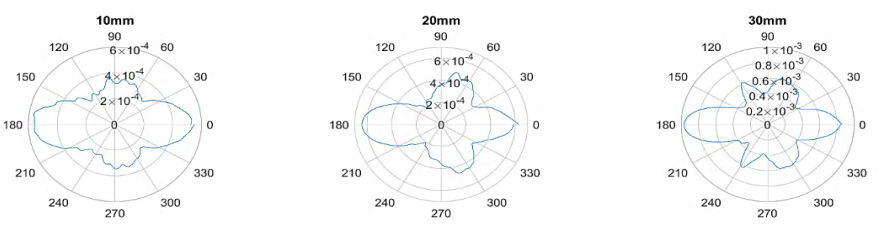

$\mathrm{Ur}_{\mathrm{r}}$
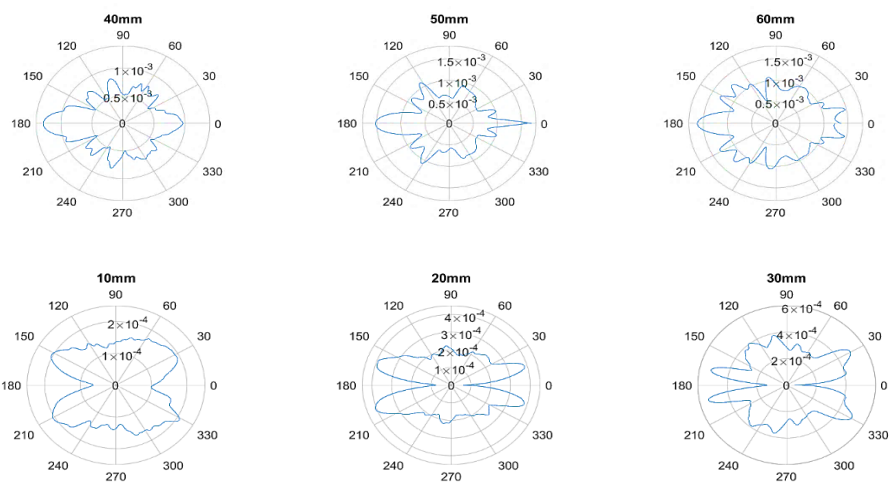

$\mathrm{U}_{\theta}$
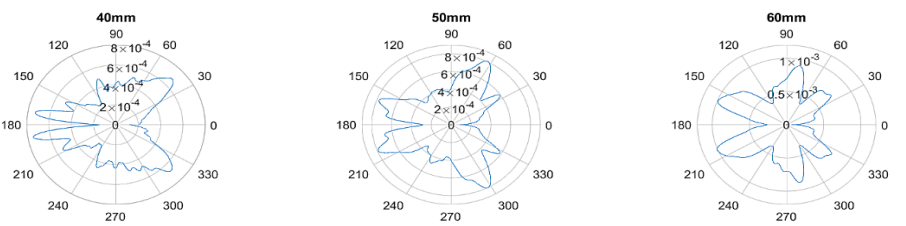

Figure 7. Scattered field amplitude directivity pattern in the $U_{r}$ direction top and $U_{\theta}$ bottom for $d$ $=(10 \mathrm{~mm}$ to $60 \mathrm{~mm})$

The scattered wave plot patterns are symmetrical along the horizontal lines in Fig 6 and Fig 7 for both $U_{r}$ and $U_{\theta}$ direction. Fig 6 demonstrates that the defects from $d=7 \mathrm{~mm}$ to $d=10 \mathrm{~mm}$ show almost an identical scatter pattern in both $\mathrm{U}_{\mathrm{r}}$ and $\mathrm{U}_{\theta}$ directions. For the $\mathrm{U}_{\mathrm{r}}$ direction the main lobe is at $180^{\circ}$ with a significant but smaller lobe at $0^{\circ}$, along with identical smaller lobes at $90^{\circ}$ and $270^{\circ}$. For the $\mathrm{U}_{\theta}$ direction the main lobes are at $30^{\circ}$ and $330^{\circ}$, with significant but smaller identical lobes at $150^{\circ}$ and $210^{\circ}$.For $\mathrm{d}=5 \mathrm{~mm}$ and $\mathrm{d}=6 \mathrm{~mm}$ a slight difference is observed in the $\mathrm{U}_{\mathrm{r}}$ direction where the main lobe is at $180^{\circ}$ with smaller lobes at $0^{\circ}, 60^{\circ}$ and $300^{\circ}$.

The observations from Fig 7. show that when the defect diameter increases in the $\mathrm{U}_{\mathrm{r}}$ direction the dominant lobe fluctuates between $0^{\circ}$ and $180^{\circ}$. At $\mathrm{d}=10 \mathrm{~mm}$ the main lobe is at $180^{\circ}$ with a significant but smaller lobe at $0^{\circ}$ along with smaller lobes at $90^{\circ}$ and $270^{\circ}$. Similarly, as $d$ is increased to $20 \mathrm{~mm}$ and $30 \mathrm{~mm}$ there is a shift in the smaller lobes by $30^{\circ}$ and $60^{\circ}$ respectively. For $\mathrm{d}=40 \mathrm{~mm}$ there are smaller lobes at $130^{\circ}, 160^{\circ}, 220^{\circ}, 250^{\circ}$ and the lobe at $0^{\circ}$ shrinks, these smaller lobes are shifted by $10^{\circ}$ upon increasing $\mathrm{d}$ to $50 \mathrm{~mm}$ while a sharp main lobe is at $0^{\circ}$. For $\mathrm{d}=60 \mathrm{~mm}$ the main lobe is at $180^{\circ}$ with smaller distinct lobes from $90^{\circ}$ to $270^{\circ}$. For the $\mathrm{U}_{\theta}$ direction the forward scatter energy is focused more along the edge as the defect size increases. For $\mathrm{d}=10 \mathrm{~mm}$ and $30 \mathrm{~mm}$ the main lobes are located at $30^{\circ}$ and $330^{\circ}$, while for $\mathrm{d}=20 \mathrm{~mm}, 40 \mathrm{~mm}$ and $50 \mathrm{~mm}$ the main lobes are located at $145^{\circ}$ and $205^{\circ}, 45^{\circ}$ and $315^{\circ}$, and $60^{\circ}$ and $300^{\circ}$ respectively. For $\mathrm{d}=10 \mathrm{~mm}$ and $30 \mathrm{~mm}$ the smaller lobes are located at $150^{\circ}$ and $210^{\circ}$, while for $\mathrm{d}=20 \mathrm{~mm}, 40 \mathrm{~mm}, 50 \mathrm{~mm}$ and $60 \mathrm{~mm}$ the smaller lobes are located at $25^{\circ}$ and $355^{\circ}, 150^{\circ}$ and $210^{\circ}$, $145^{\circ}$ and $205^{\circ}$, and $150^{\circ}$ and $210^{\circ}$ respectively.

\section{Discussion}

FE results presented in this paper showed that the scattered wave fields directivity patterns are dependent on the defect diameter where the amplitude increase with an order of magnitude. In 
the $\mathrm{U}_{\mathrm{r}}$ direction, the Fig 7 plots show that forward and back scattered regions are comparable in size with two similar lobes about the $\theta=180^{\circ}$ radial line for $d=10 \mathrm{~mm}$ to $d=40 \mathrm{~mm}$. As the defect diameter increase, the forward scatter decreases slightly, and the back scattered increases slightly becoming dominant. The same can be said when $(\lambda)>d$ for defects from $d=5 \mathrm{~mm}$ to $\mathrm{d}=9 \mathrm{~mm}$. For $\lambda \leq \mathrm{d}$, the $\mathrm{P}$ incident wave is dominant with along with a weak incident $\mathrm{S}$ wave, the scattering wavefield have a contribution from this incident $\mathrm{S}$ wave as well. The patterns are only symmetrical on the $0^{\circ}$-axis. This is due to the creeping shear wave [24], coalescing with the leaky Rayleigh wave propagating on the circumference of the defect $[25,26]$. This indicates that defect size characterisation can be related to the scattered field amplitude and incident wave mode conversion. Thus, methods of detecting and characterising defects within a solid which rely solely on the reflected wave field do not provide enough information for the characterisation of the defect size.

\section{Conclusion}

The scattered wave field of bulk stress waves in an isotropic solid cylinder with a cylindrical defect using narrow-band tone-burst excitation has been reported. This paper illustrated that the diameter of the cylindrical defect affects the scattered wave pattern and that the scattered pattern can be influenced by incident wave components $\mathrm{P}$ and $\mathrm{S}$ mode conversion. This study provides a fundamental analysis to address Teredo marine borers defects effects on wave fields. Further, this paper highlights the importance of scattered wave field analysis for defect size characterisation. Future work will address the Teredo defects in a multiple defects' scenario.

\section{References}

[1] Salmiah, K.R.U., RESISTANCE OF FIVE TIMBER SPECIES TO MARINE BORER ATTACK. Journal of Tropical Forest Science, 2015. 27 ((3)): p. 400-412

[2] Charles, F., et al., Wood decay at sea. Journal of Sea Research, 2016. 114: p. 22-25.

https://doi.org/10.1016/j.seares.2016.05.002

[3] Kelly, S.W., UNDERWATER INSPECTION CRITERIA. 1999, NAVAL FACILITIES

ENGINEERING SERVICE CENTER: Port Hueneme, California.

[4] Nowak, T.P., J. Jasieńko, and K. Hamrol-Bielecka, In situ assessment of structural timber using the resistance drilling method - Evaluation of usefulness. Construction and Building Materials, 2016. 102: p. 403-415. https://doi.org/10.1016/j.conbuildmat.2015.11.004

[5] Greenawald, E.C., et al. Underwater x-ray tomography of composite sonar domes via collimated backscatter imaging. in Nondestructive Evaluation of Materials and Composites II. 1998. International Society for Optics and Photonics. https://doi.org/10.1117/12.301510

[6] Riis, N., et al., Limited-data x-ray CT for underwater pipeline inspection. Inverse Problems, 2018. 34(3): p. 034002. https://doi.org/10.1088/1361-6420/aaa49c

[7] Wang Xiping, F.D., Crystal Pilon, Brian K. Brashaw, Robert J. Ross, and Roy F. Pellerin, Assessment of decay in standing timber using stress wave timing nondestructive evaluation tools: A guide for use and interpretation. 2004, United States Department of Agriculture Forest Service Forest Products Laboratory. https://doi.org/10.2737/FPL-GTR-147

[8] Kato, K., Reflection of sound wave due to a hollow cylinder in an elastic body. Osaka University, Memoirs of the Institute of Scientific and Industrial Research, 1952. 9: p. 16-20.

[9] White, R.M., Elastic Wave Scattering at a Cylindrical Discontinuity in a Solid. The Journal of the Acoustical Society of America, 1958. 30(8): p. 771-785. https://doi.org/10.1121/1.1909759 
[10] Lewis, T.S. and D.W. Kraft, Mode conversion relation for elastic waves scattered by a cylindrical obstacle in a solid. The Journal of the Acoustical Society of America, 1974. 56(6): p. 1899-1901. https://doi.org/10.1121/1.1903529

[11] Zhang, J., et al., Study on the Single Scattering of Elastic Waves by a Cylindrical Fiber with a Partially Imperfect Bonding Using the Collocation Point Method. Shock and Vibration, 2018. 2018: p. 114. https://doi.org/10.1155/2018/7516402

[12] Kuo, H.-Y., Y.-A. Huang, and C.-H. Sun, Scattering of anti-plane shear waves by arbitrarily distributed circular cylinders in a functionally graded multiferroic fibrous composite. Acta Mechanica, 2017. 229(4): p. 1483-1501. https://doi.org/10.1007/s00707-017-2079-X

[13] Bucur, V., Acoustics of Wood. 2nd Edition ed, ed. S.S.i.W. Science. 2006: Springer. https://doi.org/10.1007/3-540-30594-7

[14] Yan, N., Numerical Modelling and Condition Assessment of Timber Utility Poles using Stress Wave Techniques. 2015, University of Technology, Sydney.

[15] Wang, X. and L. Sudak, Scattering of elastic waves by multiple elastic circular cylinders with imperfect interface. Waves in Random and Complex Media, 2007. 17(2): p. 159-187. https://doi.org/10.1080/17455030601118376

[16] Liu, Y., Wu, R.S. and Ying, C.F, Scattering of elastic waves by an elastic or viscoelastic cylinder. Geophysical Journal International, 2000. 142(2): p. 439-460. https://doi.org/10.1046/j.1365246x.2000.00173.x

[17] Grave, B.H., Natural history of shipworm, Teredo navalis, at Woods Hole, Massachusetts. The Biological Bulletin, 1928. 55(4): p. 260-282. https://doi.org/10.2307/1537080

[18] Sikdar, S. and S. Banerjee, Guided wave propagation in a honeycomb composite sandwich structure in presence of a high density core. Ultrasonics, 2016. 71: p. 86-97.

https://doi.org/10.1016/j.ultras.2016.05.025

[19] Bingham, J. and M. Hinders, 3D elastodynamic finite integration technique simulation of guided waves in extended built-up structures containing flaws. Journal of Computational Acoustics, 2010. 18(02): p. 165-192. https://doi.org/10.1142/S0218396X10004097

[20] Courant, R., K. Friedrichs, and H. Lewy, Über die partiellen Differenzengleichungen der mathematischen Physik. Mathematische annalen, 1928. 100(1): p. 32-74.

https://doi.org/10.1007/BF01448839

[21] Leckey, C.A.C., et al., Simulation of guided-wave ultrasound propagation in composite laminates: Benchmark comparisons of numerical codes and experiment. Ultrasonics, 2018. 84: p. 187-200. https://doi.org/10.1016/j.ultras.2017.11.002

[22] Chiu, W.K., L.R.F. Rose, and B.S. Vien, Scattering of the Edge-guided Wave by an Edge Crack at a Circular Hole in an Isotropic Plate. Procedia Engineering, 2017. 188: p. 309-316.

https://doi.org/10.1016/j.proeng.2017.04.489

[23] Shull, P.J., Nondestructive evaluation: theory, techniques, and applications. 2002: CRC press. https://doi.org/10.1201/9780203911068

[24] Aldrin, J.C., et al., Scattering of obliquely incident shear waves from a cylindrical cavity. The Journal of the Acoustical Society of America, 2011. 129(6): p. 3661-3675.

https://doi.org/10.1121/1.3583540

[25] Nagy, P.B., M. Blodgett, and M. Golis, Weep hole inspection by circumferential creeping waves. NDT \& E International, 1994. 27(3): p. 131-142. https://doi.org/10.1016/0963-8695(94)90604-1

[26] Doherty, C. and W.K. Chiu, Scattering of ultrasonic-guided waves for health monitoring of fuel weep holes. Structural Health Monitoring, 2012. 11(1): p. 27-42.

https://doi.org/10.1177/1475921710395814 